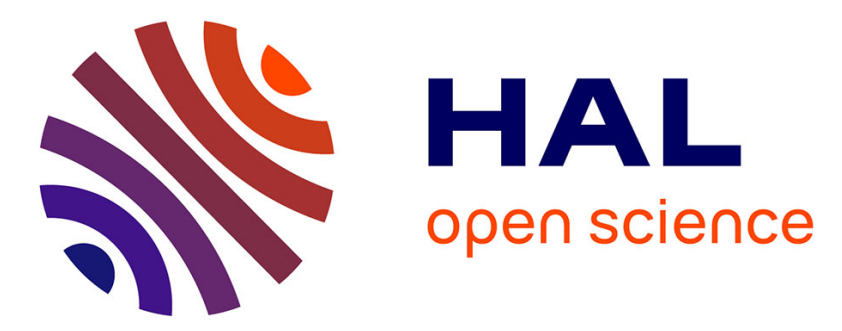

\title{
GGA+U study of uranium mononitride: A comparison of the U-ramping and occupation matrix schemes and incorporation energies of fission products
}

Antoine Claisse, Marco Klipfel, Niclas Lindbom, Michel Freyss, Pär Olsson

\section{To cite this version:}

Antoine Claisse, Marco Klipfel, Niclas Lindbom, Michel Freyss, Pär Olsson. GGA+U study of uranium mononitride: A comparison of the U-ramping and occupation matrix schemes and incorporation energies of fission products. Journal of Nuclear Materials, 2016, 478, pp.119-124. 10.1016/j.jnucmat.2016.06.007 . cea-02063633

\section{HAL Id: cea-02063633 \\ https://hal-cea.archives-ouvertes.fr/cea-02063633}

Submitted on 11 Mar 2019

HAL is a multi-disciplinary open access archive for the deposit and dissemination of scientific research documents, whether they are published or not. The documents may come from teaching and research institutions in France or abroad, or from public or private research centers.
L'archive ouverte pluridisciplinaire HAL, est destinée au dépôt et à la diffusion de documents scientifiques de niveau recherche, publiés ou non, émanant des établissements d'enseignement et de recherche français ou étrangers, des laboratoires publics ou privés. 


\title{
GGA+U study of uranium mononitride: A comparison of the U-ramping and occupation matrix schemes and incorporation energies of fission products
}

\author{
Antoine Claisse ${ }^{\text {a, * }}$, Marco Klipfel ${ }^{\text {b }}$, Niclas Lindbom ${ }^{\text {a }}$, Michel Freyss ${ }^{c}$, Pär Olsson ${ }^{a}$ \\ ${ }^{a}$ KTH Royal Institute of Technology, Reactor Physics, AlbaNova University Centre, 10691 Stockholm, Sweden \\ ${ }^{\mathrm{b}}$ European Commission, Joint Research Centre, Institute for Transuranium Elements, P.O. Box 2340, D-76125 Karlsruhe, Germany \\ ${ }^{\text {c } C E A, ~ D E N, ~ D E C, ~ C e n t r e ~ d e ~ C a d a r a c h e, ~} 13108$ Saint-Paul-lez-Durance, France
}

\section{H I G H L I G H T S}

- We studied bulk uranium nitride with means of DFT $+\mathrm{U}$ with the U-ramping scheme and the OMC scheme.

- We produces a density of states plot and calculated the elastic constants of UN.

- We calculated the incorporation energy of many fission products in UN, and corrected them to take into account the elastic interactions.

- The OMC scheme should be used over the U-ramping scheme.

- Fission products go to larger crystal sites.

\section{A R T I C L E I N F O}

\section{Article history:}

Received 15 March 2016

Received in revised form 1 June 2016

Accepted 2 June 2016

Available online 6 June 2016

\begin{abstract}
A B S T R A C T
Uranium mononitride is studied in the DFT $+\mathrm{U}$ framework. Its ground state is investigated and a study of the incorporation of diverse fission products in the crystal is conducted. The U-ramping and occupation matrix control (OMC) schemes are used to eliminate metastable states. Beyond a certain amount of introduced correlation, the OMC scheme starts to find a lower total energy. The OMC scheme is chosen for the second part of this study. Furthermore, the influence of the magnetic ordering is studied using the U-ramping method, showing that antiferromagnetic order is the most stable one when the U parameter is larger than $1.75 \mathrm{eV}$. The effect on the density of states is investigated and elastic constants are provided for comparison with other methods and experiments. The incorporation energies of fission products in different defect configurations are calculated and these energies are corrected to take into account the limited size of the supercell.
\end{abstract}

(c) 2016 Elsevier B.V. All rights reserved.

\section{Introduction}

With the regained interest in innovative nuclear fuels for next generation reactors and in accident-tolerant fuel programs for current reactors, as well as the advent of more sophisticated simulation techniques and improved hardware, the number of theoretical studies on advanced nuclear fuels is increasing. Indeed, fuels such as UN have a higher fissile density allowing for a longer operation time, a higher thermal conductivity giving larger safety margins and have been shown to be a better candidate to burn

\footnotetext{
* Corresponding author.

E-mail address: claisse@kth.se (A. Claisse).
}

long-lived minor actinides [1].

For many years, density functional theory [2,3] (DFT) calculations have been extensively applied for the modeling of bulk properties of actinide compounds [4-9]. However, the description of the electronic structure of actinides and their compounds is complicated by the localisation-delocalisation behavior of the $5 f$ electrons, and the challenges in describing $5 f$ elements in the solid state remain significant. The so-called DFT $+U$ approach developed by Anisimov's group [10-12] following the idea of Hubbard [13,14] and implemented in electronic structure codes by for instance Dudarev et al. [15] or Lichtenstein et al. [16] is capable of improving the description of strong correlation, leading to a better prediction of band gaps, densities of states and electronic and magnetic properties [4,17-19] among others. 
A recent review on the various computational approximations to deal with strong correlations in actinide oxides was carried out by Wen et al. [20], in which a paragraph was devoted to simulations of uranium nitride. This review was complemented later [21] to give more emphasis on the results and it was concluded that the DFT + U scheme is indeed needed, although using the computationally more expensive HSE hybrid functional may provide similar results. However, the orbital anisotropy induced by the improved modeling of electron correlation by DFT $+U$ increases the number of metastable states (i.e. the number of local energy minima on the potential energy surface) [22]. DFT + U calculations are prone to converge to these metastable states depending on the initial conditions, a problem that is shared with the HSE hybrid functionals [23]. A comparison of formation energies of $\mathrm{UO}_{2}$ by different authors was reported by Dorado et al. [24], showing extreme variations. It is consequently essential to ensure that the ground state of the system has indeed been reached to obtain the correct description of the bulk material properties. This is needed before one can attempt to study point defects and impurities.

Several approaches have been used so far to find the ground state among these many possible metastable states within DFT $+\mathrm{U}$ modeling. The so-called "U-ramping" approach [22,25] uses a gradual increase of the Hubbard $U$ and exchange $J$ parameters from zero to their final values. The starting point of each simulation is the final point of the previous one. Such a U-ramping has to be done for every calculation and this scheme is therefore becoming very computationally expensive when many simulations are needed, such as for studying point defects. Recently Rabone and Krack [26] presented a procedure for bypassing metastable states by using an f-occupation smearing combined with U-ramping, which was applied to uranium dioxide surfaces.

The quasi annealing approach [27] uses a random added electronic noise which is slowly turned off to allow the system to converge to the ground state. This noise is acting upon the minimization path to help avoiding the metastable states.

The controlled symmetry reduction (CSR) method [17] is based on a geometric distortion which ensures the convergence of the system to a specific state. By reviewing experimental data on for instance the magnetic ordering, it is possible to gain knowledge about the shape and deformation of the primary cell. The starting point is then an exaggeratedly deformed cell for which there will be no metastable state, and the distortion will be slowly reduced in successive simulations where the final electronic density found by a simulation is used as an initial condition for the next one. This has been applied to UN with promising results by Gryaznov et al. [28].

Another procedure to reach unequivocally the ground state was presented by Jomard et al. [29] on plutonium oxides. The occupation matrix control scheme (OMC) is based on the monitoring of the occupation matrices of the correlated orbitals. By imposing an initial occupation matrix it is possible to control the filling of the $5 f$ levels. The imposed occupation matrices are defined according to the quantum numbers $\mathrm{m}_{\mathrm{i}}$ and $\mathrm{m}_{\mathrm{j}}$ corresponding to the orbitals which are filled.

In the present work we demonstrate the applicability of the occupation matrix control scheme to uranium nitride, by comparing it with U-ramping results. In the first part of this paper, the influence of magnetism is investigated using the U-ramping method. Then, a systematic search of the ground-state occupation matrix is carried out. The electronic ground state and metastable states are addressed and characterized by comparing structural and magnetic properties and the density of states (DOS). The capability of the OMC method to find the ground state is discussed, and once it is established, its applicability to point defect calculations is assessed by studying the incorporation of fission products in the UN crystal. This has already been done using conventional DFT in UN
[30,31], in UC [32] and in $\mathrm{UO}_{2}$ [33]. However, such a systematic study using the DFT + U framework in UN is still missing. One could also mention that this study should be further improved taking into account the spin-orbit coupling (SOC), since it has been shown by Gryaznov et al. that its effect should not be neglected [28]. However, all of the simulations presented in this study have been done without SOC, as a first step.

\section{Computational details}

All the calculations done in this work are carried out within the density functional theory framework [2,3] using the projector augmented wave (PAW) formalism [34] as implemented in the code VASP (Vienna Ab initio Simulation Package) [35-37]. For the Hubbard parameter $U$, the rotationally invariant Liechtenstein [16] approach was chosen, and the values $U$ and $\mathrm{J}$ were fixed to $2.0 \mathrm{eV}$ and $0.1 \mathrm{eV}$, respectively $[18,28]$. One should realize that this choice is not to be seen as a fitting parameter, and ideally, $U$ would come from an experiment or another simulation, and not from fitting the room temperature lattice parameter or magnetic moment on a zero-Kelvin simulation. Using the U-ramping scheme, the U-value was increased from 0 to $2.5 \mathrm{eV}$ in steps of $0.1 \mathrm{eV}$ to study the magnetic stability. In order to study the appearance of the metastable states using the OMC framework, the full exploration was carried out for different values of $U$. The integration in the reciprocal space over the Brillouin zone was performed using $9 \times 9 \times 9$ Monkhorst-Pack meshes [38] for the antiferromagnetic cell with 8 atoms. For the 64 -atom supercells, a $2 \times 2 \times 2 \mathrm{k}$-point mesh has been used after comparing the results with a $5 \times 5 \times 5$ k-point sampling for the incorporation energies in a few cases, yielding a difference of under $4 \%$. The cut-off energy for the plane wave expansion was fixed at $600 \mathrm{eV}$ and the fractional electron occupancies were estimated with the method of Methfessel and Paxton [39] with a smearing parameter of $0.1 \mathrm{eV}$. Bulk calculations are done using UN unit cells with 8 atoms, with and without crystal symmetry. Simulations in a 64-atom cell have been performed without any imposed crystal symmetry and otherwise identical parameters for the point defect configurations. In Fig. 1 the antiferromagnetic ordering (type 1 ) of the 8 -atom UN cell is shown, as found by Curry [40].

In order to determine the ground state with the OMC scheme, different occupation matrices were imposed at the beginning of

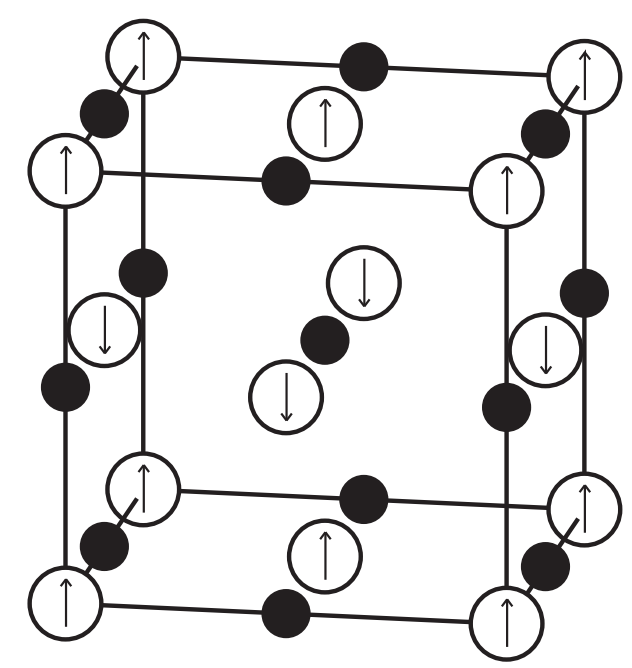

Fig. 1. UN rock-salt crystal structure showing antiferromagnetic order. White atoms are uranium while black atoms correspond to nitrogen atoms. Arrows represent the spin orientation. 
each calculation. Each occupation matrix corresponds to a particular filling of the uranium $5 f$-orbitals. We only impose diagonal occupation matrices leading to 21 different ways of filling the seven $5 f$-orbitals with two correlated valence $f$-electrons with full occupancy [41,42]. Each of the 21 ways is called an electronic configuration. Since there are several degenerate $f$ levels, some of the electronic configurations are identical by symmetry i.e. they have identical electronic energies. In each calculation, one particular diagonal occupation matrix is imposed during the first 15 steps of the first self-consistent cycle. This constraint is then lifted and the calculation is left to converge without further constraints. Further details about the OMC scheme are described elsewhere [24,29].

The incorporation energy of an impurity is calculated as follows:

$E_{\text {inc }}=E_{\text {cell }}-E_{\text {cell }}^{\text {ref }}-E_{\text {coh }}$

where $E_{\text {inc }}$ is the incorporation energy, $E_{\text {cell }}$ is the total energy of the cell including the impurity, $E_{\text {cell }}^{\text {ref }}$ is the total energy of the cell with the defect but without the impurity and $\mathrm{E}_{\mathrm{coh}}$ is the cohesive energy of the fission products.

The cohesive energy of the fission products is computed considering the room temperature phase. For instance, for xenon, we used a gas atom, i.e. a single atom in a large box, and for solids, we used a unit cell of its crystallographic form and divided the total energy by the number of atoms.

\section{Results}

Standard energy calculations of $5 f$-electron systems using the $\mathrm{GGA}+\mathrm{U}$ technique are likely to be trapped in local energy minima on the potential energy surface instead of reaching the energetic ground state. For instance free relaxation of the UN crystal in its experimental geometry leads to a total energy which is higher than the one of the ground state found with the OMC, by $5 \mathrm{meV}$ per $U$ atom for a U-value of $2.0 \mathrm{eV}$. The next two subsections deal with proper characterization of the ground state.

\subsection{U-ramping scheme on bulk UN}

In this part, the influence of magnetism on the crystal stability is investigated. The cell size, shape and the ionic positions have been allowed to relax. The results are displayed in Fig. 2.

As has been previously shown [28], without the correlation term, the stable phase at $0 \mathrm{~K}$ is the ferromagnetic (FM) one. When

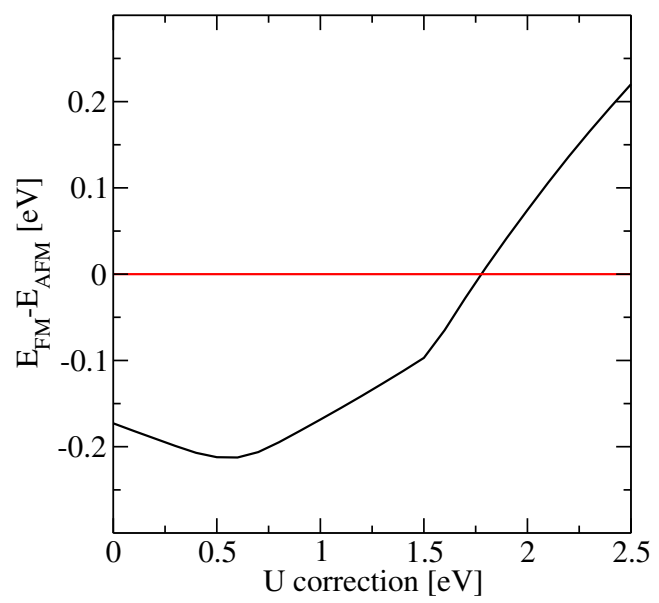

Fig. 2. Relative energy of the FM and AFM states of UN as a function of the $U$ parameter. the $\mathrm{U}$ term reaches around $1.75 \mathrm{eV}$, the most stable phase becomes antiferromagnetic (AFM), which is in line with low-temperature experiments [40]. This effect is surprisingly not observed when the same calculations are carried out with the spin-orbit coupling taken into account. Gryaznov et al. [28] concluded that the effect of the spin-orbit coupling is important in their study and more simulations are still needed to corroborate this finding.

We noted no effect of the U-ramping scheme for U-parameters up to $2.5 \mathrm{eV}$. A single calculation with a U-parameter of $3.5 \mathrm{eV}$ yielded a higher total energy than the U-ramping method, but such a high $U$-value is not realistic and higher than any suggested value for UN in the literature. That seems to indicate that the benefits of using the U-ramping scheme for UN are not apparent, at least when the SOC is not considered.

In addition, the authors of the U-ramping recognize that this method can yield a higher ground state energy as compared with the OMC framework [43].

\subsection{Occupation matrix control scheme on bulk UN}

\subsubsection{Relaxation and total energy}

Table 1 shows the initial diagonal occupation matrices for UN and the relative energies computed for different U-values. For each electronic initial state, the difference in energy relative to the lowest-energy state is given in $\mathrm{meV}$. The calculations have been performed in an 8-atom cell. Whereas the energy differences have been reported for 5 different values of $U$, the lattice constants and magnetic moments are indicated for the free relaxation case with a $\mathrm{U}$-value of $2.0 \mathrm{eV}$. J has been kept equal to $0.1 \mathrm{eV}$ for all the simulations. Generally, no crystal symmetry constraints were imposed, except for one case, indicated in Table 1 by SYM, where the conventional computation time reduction through symmetry conservation was used. This one case was selected since $2 \mathrm{eV}$ of added correlation is the value commonly accepted in the literature, to show the importance of turning off the symmetries.

It can be noted that the metastable states seem to appear only after a certain amount of introduced correlation. They are completely absent at $\mathrm{U}=0.5 \mathrm{eV}$; there are five different states for $\mathrm{U}=2.0 \mathrm{eV}$ and eight for $\mathrm{U}=2.5 \mathrm{eV}$. Metastable states of higher energy can be found when the crystal symmetries are conserved, which should be expected and is behind the reasoning for the CSR scheme. For computations not conserving crystal symmetry, eight occupation matrices converged to the electronic ground state (GS) for the chosen value of $U$, having slight cell distortion. Gryaznov et al. have shown that the cell is not cubic anymore when U-J is above $1 \mathrm{eV}$, and that for the AFM phase without SOC, the c/a ratio is below 1 [28], whereas Lu et al. have found a ratio above unity [18]. In this study, the tetragonal shape seems not to be conserved as soon as the symmetry constraints are lifted, in favor of a orthorhombic phase. It can be noted that the second metastable state, $60 \mathrm{meV}$ per $\mathrm{U}$ atom above the ground state yields a tetragonal structure. Experimentally, no tetragonal distortion has been noted (perfect cubic cell with $\mathrm{a}=4.888 \AA$ ) [44], although it could be expected at low temperature due to the first-order antiferromagnetism. The authors have no explanation for the orthorhombicity. The magnetic moment is found to be similar to previous results without SOC [28].

Comparing the ground state energy found by using the OMC method and the U-ramping approach (Fig. 3), one can see that up to a U-value of $2.0 \mathrm{eV}$, the difference seems to be limited. However, when $U$ becomes larger, the OMC method gives an obviously lowerenergy state. Looking precisely at the numbers, already for an added correlation of $0.5 \mathrm{eV}$, the OMC framework yields a lower total energy than the $U$-ramping ( $4 \mathrm{meV}$ per $\mathrm{U}$ atom for $\mathrm{U}=2.0 \mathrm{eV}$ ), and the latter should not be trusted to yield the ground state. 
Table 1

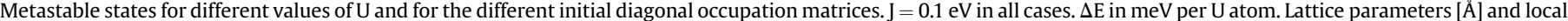

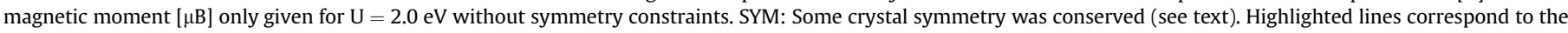
ground-state configurations for $\mathrm{U}=2.0 \mathrm{eV}$ without symmetry constraints.

\begin{tabular}{|c|c|c|c|c|c|c|c|c|c|c|}
\hline Initial matrix & $\Delta \mathrm{E} \mathrm{U}=0.5 \mathrm{eV}$ & $\Delta \mathrm{E} \mathrm{U}=1.0 \mathrm{eV}$ & $\Delta \mathrm{E} \mathrm{U}=1.5 \mathrm{eV}$ & $\Delta \mathrm{E} S Y M \quad U=2.0 \mathrm{eV}$ & $\Delta \mathrm{E} \mathrm{U}=2.0 \mathrm{eV}$ & $\Delta \mathrm{E} \mathrm{U}=2.5 \mathrm{eV}$ & $\mathrm{a}$ & b & c & $\mu$ \\
\hline 0000011 & 0 & 33 & 36 & 0 & 60 & 32 & 4.96 & 5.07 & 4.96 & 1.95 \\
\hline 0000101 & 0 & 33 & 36 & 0 & 66 & 130 & 4.83 & 4.98 & 4.97 & 1.54 \\
\hline 0001001 & 0 & 0 & 94 & 162 & 60 & 105 & 4.96 & 5.07 & 4.96 & 1.95 \\
\hline 0010001 & 0 & 0 & 0 & 5 & 0 & 0 & 4.90 & 4.97 & 5.04 & 1.89 \\
\hline 0100001 & 0 & 33 & 36 & 375 & 66 & 150 & 4.83 & 4.98 & 4.97 & 1.54 \\
\hline 1000001 & 0 & 0 & 0 & 5 & 0 & 0 & 4.90 & 4.97 & 5.03 & 1.89 \\
\hline 0000110 & 0 & 48 & 82 & 0 & 40 & 32 & 4.96 & 5.03 & 4.90 & 1.82 \\
\hline 0001010 & 0 & 33 & 36 & 0 & 66 & 130 & 4.97 & 4.98 & 4.83 & 1.54 \\
\hline 0010010 & 0 & 0 & 0 & 5 & 0 & 0 & 5.03 & 4.97 & 4.90 & 1.89 \\
\hline 0100010 & 0 & 33 & 36 & 0 & 66 & 130 & 4.97 & 4.98 & 4.83 & 1.54 \\
\hline 1000010 & 0 & 0 & 0 & 5 & 0 & 0 & 5.03 & 4.97 & 4.90 & 1.89 \\
\hline 0001100 & 0 & 33 & 36 & 0 & 66 & 32 & 4.83 & 4.98 & 4.97 & 1.54 \\
\hline 0010100 & 0 & 0 & 0 & 5 & 0 & 0 & 4.90 & 4.97 & 5.03 & 1.89 \\
\hline 0100100 & 0 & 33 & 36 & 207 & 66 & 134 & 4.97 & 4.98 & 4.83 & 1.54 \\
\hline 1000100 & 0 & 0 & 0 & 5 & 0 & 0 & 4.90 & 4.97 & 5.03 & 1.89 \\
\hline 0011000 & 0 & 0 & 0 & 5 & 0 & 0 & 5.03 & 4.97 & 4.90 & 1.89 \\
\hline 0101000 & 0 & 19 & 36 & 162 & 66 & 192 & 4.97 & 4.98 & 4.83 & 1.54 \\
\hline 1001000 & 0 & 0 & 0 & 5 & 0 & 202 & 5.03 & 4.97 & 4.90 & 1.89 \\
\hline 0110000 & 0 & 0 & 28 & 5 & 65 & 170 & 4.96 & 4.86 & 4.96 & 1.65 \\
\hline 1010000 & 0 & 4 & 28 & 5 & 65 & 170 & 4.96 & 4.86 & 4.96 & 1.65 \\
\hline 1100000 & 0 & 0 & 15 & 5 & 65 & 170 & 4.96 & 4.86 & 4.96 & 1.65 \\
\hline $\operatorname{Exp}[40,45]$ & - & - & - & - & - & - & 4.886 & 4.886 & 4.886 & 0.75 \\
\hline
\end{tabular}

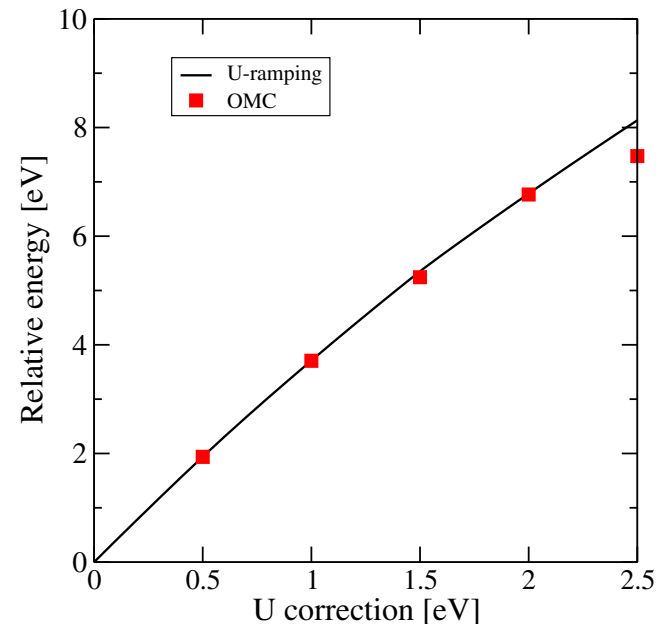

Fig. 3. Comparison of the total energy obtained using the U-ramping and OMC schemes. The energy reference is taken as the energy of the system without a Ucorrection.

From this point, all the results presented have been achieved using the OMC method.

\subsubsection{Density of states}

The density of states (Fig. 4) is very similar to what has been found elsewhere, using either the CSR scheme [28], no scheme [18], or the more advanced framework of dynamical mean field theory [41]. Unfortunately, it is not possible to compare directly with the study of Sun [46], using the spin-polarized T-matrix fluctuation exchange solver, since only the paramagnetic and ferromagnetic phases were investigated there.

As expected, the introduced correlation is sufficient to split the $f$ band and separate the $\mathrm{f}^{5 / 2}$ and $\mathrm{f}^{7 / 2}$ contributions, while conserving a metallic behavior. Without this correlation, there is no splitting [21].

\subsubsection{Elastic properties}

The elastic properties of this orthorhombic system have been

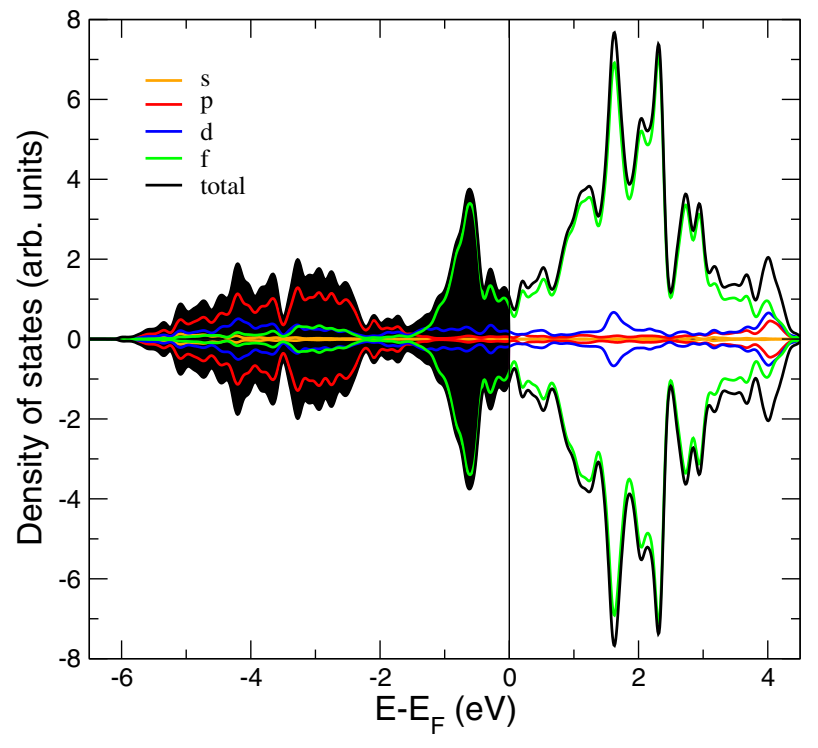

Fig. 4. Density of states of UN.

computed using the small displacement method to get the Hessian matrix. The coefficients of the Hessian matrix are then used to calculate the elastic constants. A bulk modulus of $165 \mathrm{GPa}$, a Young's modulus of $236 \mathrm{GPa}$ and a shear modulus of $94 \mathrm{GPa}$ have been determined. This compares reasonably well with the experiments reviewed by Hayes et al. [47] of respectively $185 \mathrm{GPa}$, $258 \mathrm{GPa}$ and $111 \mathrm{GPa}$ for a fully-dense sample at room temperature although it is a bit closer to the results corresponding to a porosity of few percent, which is not present in the perfect cell used for this work. Comparing with previous numerical simulations [18,48], the bulk modulus found is about $15 \%$ lower. This could be explained by the lack of a scheme to avoid the metastable states in these early works, a different choice of the couple $(\mathrm{U}, \mathrm{J})$, or even the fact that we find an orthorhombic cell compared to a tetragonal distortion in Ref. [18]. As a quick check, the elastic constants have been 
computed on a tetragonal metastable state obtained with the initial occupation matrix (0000011), but yielding a bulk modulus of $159 \mathrm{GPa}$, similar to the one of the ground state. Thus, metastable states only affect the elastic properties by a few percent.

\subsection{Incorporation energies of fission products}

The incorporation energies of the most common fission products have been calculated in the UN crystal. Four positions have been considered, similarly to what had been done using conventional DFT in UN [30] and in UC [32]. These atoms have been located in both uranium and nitrogen substitutional positions, in a Schottky defect and in interstitial positions (with reduced coordinates (0.25.0.25.0.25) or (0.25.0.25.0.50), whichever was more stable), as indicated in the table. No split interstitial configurations were observed. Considering the high packing fraction of the rocksalt structure, it is not surprising that the incorporation energies in the interstitial position are systematically higher than in the other positions. The $\mathrm{N}$ vacancy also seems to be avoided by these elements, but the incorporation energy in a Schottky defect or in a uranium vacancy are usually very similar, and the most stable of the two depends on the impurity.

The incorporation energies of the fission products indicate where they will preferentially be located in the crystal, and therefore which diffusion paths they may take. Their diffusion coefficients will however most likely be determined by different diffusion mechanisms. It might be worth noting that the incorporation energy does not take into account the formation energy of the occupied defect, and that for this reason, the actual location of the fission product might be different.

It is not straightforward to compare the results with previous ones since the cohesive energy of the chosen impurity reference state can vary from paper to paper, although the most stable position is unaffected. There are many similarities with GGA results, but with some differences. For instance, barium is now found more stable in a uranium vacancy, whereas it had been previously found to prefer a Schottky defect [30].

\subsubsection{Elastic correction}

Due to computational limitations, it is impossible to have a supercell big enough so that all the elastic interactions created by the incorporation of a foreign atom vanish through relaxation. This might create a bias on the calculated incorporation energies, presented in Table 2. At least two different approaches have been developed to deal with this issue: the one introduced by Varvenne et al. [49] has been adopted in this work, as the one described by Simonovic et al. [50] is restricted to isotropic media only.

This method uses the elastic properties, through the Voigt matrix, the crystal structure of the bulk and the residual stresses after the incorporation of a foreign atom to calculate the energy due to the elastic strains and the effects of the periodicity. The mathematical derivation is given in Ref. [49].

The corrected incorporation energies are presented in Table 2. One can see that the biggest changes (up to $1.5 \mathrm{eV}$ ) are present for the interstitial positions. For the most stable positions, namely the uranium vacancy and the Schottky configurations, the difference is limited to up to $0.15 \mathrm{eV}$ whereas for the nitrogen vacancy configurations, it can go up to $0.5 \mathrm{eV}$. Indeed, as previously mentioned, the rocksalt crystal lattice is a highly packed structure and adding an atom in the small spaces available will cause significant distortions, leading to great elastic strains. However, that does not change the general trend that the considered fission products are more stable in Schottky defects or in uranium vacancies (the latter is only the case for $\mathrm{He}, \mathrm{Zr}$ and $\mathrm{Ba}$ ). The small correction needed for these positions proves that our supercell is sufficiently large. The mostly
Table 2

Incorporation energy $[\mathrm{eV}]$ of fission products in $\mathrm{UN}$, with and without elastic correction (Sec 3.3.1). The preferred sites are in bold font.

\begin{tabular}{lrrrr}
\hline Position & Sub. U & Sub. N & Schottky & Inter \\
\hline He & $\mathbf{0 . 3 2}$ & 2.19 & 0.39 & 2.98 \\
Corrected & $\mathbf{0 . 3 3}$ & 2.19 & 0.39 & 2.95 \\
Kr & 2.82 & 6.11 & $\mathbf{2 . 1 3}$ & 11.43 \\
Corrected & 2.73 & 5.88 & $\mathbf{2 . 0 6}$ & 10.61 \\
Xe & 3.74 & 8.45 & $\mathbf{2 . 7 9}$ & 14.64 \\
Corrected & 3.61 & 7.90 & $\mathbf{2 . 6 4}$ & 13.18 \\
Zr & $-\mathbf{3 . 3 6}$ & -2.62 & -3.18 & 7.93 \\
Corrected & $-\mathbf{3 . 3 6}$ & -2.73 & -3.19 & 7.45 \\
I & -0.95 & 2.31 & $-\mathbf{2 . 2 4}$ & $6.76^{*}$ \\
Corrected & -1.06 & 1.80 & $-\mathbf{2 . 3 7}$ & $5.75^{*}$ \\
Br & -1.92 & -1.06 & $-\mathbf{3 . 4 8}$ & 5.64 \\
Corrected & -2.00 & -1.29 & $-\mathbf{3 . 5 7}$ & 4.82 \\
Cs & - & 6.98 & $\mathbf{0 . 8 6}$ & 12.34 \\
Corrected & - & 6.50 & $\mathbf{0 . 7 7}$ & 11.05 \\
Ba & $-\mathbf{1 . 2 1}$ & 6.33 & -1.13 & 10.85 \\
Corrected & $-\mathbf{1 . 2 8}$ & 5.90 & -1.19 & 9.59 \\
Mo & -0.49 & 3.26 & $-\mathbf{0 . 8 4}$ & $6.55^{\mathrm{a}}$ \\
Corrected & -0.52 & 3.13 & $\mathbf{0 . 9 5}$ & $6.22^{\mathrm{a}}$ \\
Tc & 0.11 & 1.88 & $-\mathbf{0 . 4 9}$ & $5.46^{\mathrm{a}}$ \\
Corrected & 0.10 & 1.73 & $-\mathbf{0 . 5 7}$ & $5.19^{\mathrm{a}}$ \\
Ru & -0.44 & 0.73 & $\mathbf{- 0 . 9 3}$ & $4.53^{\mathrm{a}}$ \\
Corrected & -0.42 & 0.54 & $\mathbf{0 . 9 6}$ & $4.16^{\mathrm{a}}$ \\
Rh & -0.22 & 1.07 & $\mathbf{- 1 . 1 3}$ & $5.01^{\mathrm{a}}$ \\
Corrected & -0.23 & 0.81 & $\mathbf{- 1 . 1 5}$ & $4.60^{\mathrm{a}}$ \\
\hline a & & & & \\
\hline
\end{tabular}

${ }^{a}$ Refers to a $(0.25,0.25,0.50)$ site. The other interstitial sites are $(0.25,0.25,0.25)$.

similar incorporation energies in these two configurations ( $U$ vacancy and Schottky defect) can be expected since the nitrogen vacancy in the Schottky defect has a smaller impact in comparison with the uranium vacancy.

\section{Conclusions and discussion}

We aimed to provide a comparison of the efficiency of the Uramping and OMC schemes for the uranium mononitride compound. It appears that the occupation matrix exploration yields a lower energy, thus closer to the ground state, than the U-ramping approach does, noticeably at least for larger introduced correlations. The U-ramping scheme does not seem to help when compared to a traditional DFT $+\mathrm{U}$ simulation at least for usual values of $U$ for UN without SOC. Considering this and the lower computational effort once the initial 21 cases have been converged, we recommend that the OMC scheme should be favored over the Uramping scheme for DFT + U UN calculations. A definite strength is that the OMC method provides a systematic scan of all possible occupation matrices ensuring that the obtained ground state corresponds to the ground state of the system.

The shape of the cell yielding the lowest energy is found to be orthorhombic instead of tetragonal as in previous studies. The magnetic moment and lattice parameters agree well with the literature.

The DOS reveals a metallic behavior with a splitting of the $f$ electron energies in two groups. The elastic constants are predicted within reasonable agreement with experiments.

The incorporation energies of many fission products have been computed in four different lattice positions. These energies have been corrected to take into account the elastic strains. The fission products are found more stable in Schottky defects or in uranium vacancies.

The influence of the spin-orbit coupling remains uncertain. Although differences have to be expected, as has been shown [28], it is unclear whether or not they will be significant for the point defect energetics. In conclusion, the OMC framework should be used in subsequent studies to determine diffusion coefficients and 
other properties of interest for reactor applications.

\section{Acknowledgements}

The authors wish to acknowledge Boris Dorado at CEA/DAM, and Emerson Vathonne and Marjorie Bertolus at CEA/DEN for the development of a VASP version supporting the OMC framework. Paul Van-Uffelen and Rudy Konings are acknowledged for proofreading. Financial support from SKB is acknowledged.

\section{References}

[1] Y. Zhang, J. Wallenius, M. Jolkkonen, Transmutation of americium in a large sized sodium-cooled fast reactor loaded with nitride fuel, Ann. Nucl. Energy 53 (2013) 26-34.

[2] P. Hohenberg, W. Kohn, Inhomogeneous electron gas, Phys. Rev. 136 (3B) (1964) B864.

[3] W. Kohn, L.J. Sham, Self-consistent equations including exchange and correlation effects, Phys. Rev. 140 (4A) (1965) A1133.

[4] R. Ducher, R. Dubourg, M. Barrachin, A. Pasturel, First-principles study of defect behavior in irradiated uranium monocarbide, Phys. Rev. B 83 (10) (2011) 104107.

[5] R.A. Evarestov, A.V. Bandura, M. Losev, E. Kotomin, Y.F. Zhukovskii, D. Bocharov, A first-principles DFT study of un bulk and (001) surface: comparative LCAO and PW calculations, J. Comput. Chem. 29 (13) (2008) 2079-2087.

[6] R. Evarestov, M. Losev, A. Panin, N. Mosyagin, A. Titov, Electronic structure of crystalline uranium nitride: LCAO DFT calculations, Phys. Status Solidi B 245 (1) (2008) 114-122.

[7] M. Freyss, First-principles study of uranium carbide: accommodation of point defects and of helium, xenon, and oxygen impurities, Phys. Rev. B 81 (1) (2010) 014101.

[8] M. Freyss, T. Petit, J.-P. Crocombette, Point defects in uranium dioxide: ab initio pseudopotential approach in the generalized gradient approximation, J. Nucl. Mater. 347 (1) (2005) 44-51.

[9] E. Kotomin, R. Grimes, Y. Mastrikov, N. Ashley, Atomic scale dft simulations of point defects in uranium nitride, J. Phys. Condens. Matter 19 (10) (2007) 106208.

[10] V.I. Anisimov, J. Zaanen, O.K. Andersen, Band theory and mott insulators: hubbard U instead of stoner I, Phys. Rev. B 44 (3) (1991) 943.

[11] V. Anisimov, I. Solovyev, M. Korotin, M. Czyżyk, G. Sawatzky, Density-functional theory and NiO photoemission spectra, Phys. Rev. B 48 (23) (1993) 16929.

[12] I. Solovyev, P. Dederichs, V. Anisimov, Corrected atomic limit in the localdensity approximation and the electronic structure of d impurities in Rb, Phys. Rev. B 50 (23) (1994) 16861.

[13] J. Hubbard, Electron correlations in narrow energy bands, in: Proceedings of the Royal Society of London A: Mathematical, Physical and Engineering Sciences, 276, The Royal Society, 1963, pp. 238-257.

[14] J. Hubbard, Electron correlations in narrow energy bands. IV. The atomic representation, in: Proceedings of the Royal Society of London A: Mathematical, Physical and Engineering Sciences, 285, The Royal Society, 1965, pp. $542-560$.

[15] S. Dudarev, G. Botton, S. Savrasov, C. Humphreys, A. Sutton, Electron-energyloss spectra and the structural stability of nickel oxide: an LSDA $+U$ study, Phys. Rev. B 57 (3) (1998) 1505.

[16] A. Liechtenstein, V. Anisimov, J. Zaanen, Density-functional theory and strong interactions: orbital ordering in mott-hubbard insulators, Phys. Rev. B 52 (8) (1995) R5467.

[17] D. Gryaznov, E. Heifets, D. Sedmidubsky, Density functional theory calculations on magnetic properties of actinide compounds, Phys. Chem. Chem. Phys. 12 (38) (2010) 12273-12278.

[18] Y. Lu, B.-T. Wang, R.-W. Li, H.-L. Shi, P. Zhang, Structural, electronic, mechanical, and thermodynamic properties of UN2: systematic density functional calculations, J. Nucl. Mater. 410 (1) (2011) 46-51.

[19] P.F. Weck, E. Kim, N. Balakrishnan, F. Poineau, C.B. Yeamans, K.R. Czerwinski, First-principles study of single-crystal uranium mono-and dinitride, Chem. Phys. Lett. 443 (1) (2007) 82-86.

[20] X.-D. Wen, R.L. Martin, T.M. Henderson, G.E. Scuseria, Density functional theory studies of the electronic structure of solid state actinide oxides, Chem. Rev. 113 (2) (2012) 1063-1096.

[21] X.-D. Wen, R.L. Martin, G.E. Scuseria, S.P. Rudin, E.R. Batista, A screened hybrid dft study of actinide oxides, nitrides, and carbides, J. Phys. Chem. C 117 (25) (2013) 13122-13128.
[22] B. Meredig, A. Thompson, H. Hansen, C. Wolverton, A. Van de Walle, Method for locating low-energy solutions within DFT + U, Phys. Rev. B 82 (19) (2010) 195128.

[23] F. Jollet, G. Jomard, B. Amadon, J. Crocombette, D. Torumba, Hybrid functional for correlated electrons in the projector augmented-wave formalism: study of multiple minima for actinide oxides, Phys. Rev. B 80 (23) (2009) 235109.

[24] B. Dorado, B. Amadon, M. Freyss, M. Bertolus, DFT $+U$ calculations of the ground state and metastable states of uranium dioxide, Phys. Rev. B 79 (23) (2009) 235125.

[25] B. Dorado, M. Freyss, B. Amadon, M. Bertolus, G. Jomard, P. Garcia, Advances in first-principles modelling of point defects in uo2: $\mathrm{f}$ electron correlations and the issue of local energy? minima, J. Phys. Condens. Matter 25 (33) (2013) 333201.

[26] J. Rabone, M. Krack, A procedure for bypassing metastable states in local basis set $D F T+U$ calculations and its application to uranium dioxide surfaces, Comput. Mater. Sci. 71 (2013) 157-164.

[27] H.Y. Geng, Y. Chen, Y. Kaneta, M. Kinoshita, Structural behavior of uranium dioxide under pressure by LSDA+U calculations, Phys. Rev. B 75 (5) (2007) 054111.

[28] D. Gryaznov, E. Heifets, E. Kotomin, The first-principles treatment of the electron-correlation and spin-orbital effects in uranium mononitride nuclear fuels, Phys. Chem. Chem. Phys. 14 (13) (2012) 4482-4490.

[29] G. Jomard, B. Amadon, F. Bottin, M. Torrent, Structural, thermodynamic, and electronic properties of plutonium oxides from first principles, Phys. Rev. B 78 (7) (2008) 075125.

[30] M. Klipfel, V.D. Marcello, A. Schubert, J. van de Laar, P.V. Uffelen, Towards a multiscale approach for assessing fission product behaviour in UN, J. Nucl. Mater. 442 (13) (2013) 253-261.

[31] M. Klipfel, P. Van Uffelen, Ab initio modelling of volatile fission products in uranium mononitride, J. Nucl. Mater. 422 (1) (2012) 137-142.

[32] É. Bévillon, R. Ducher, M. Barrachin, R. Dubourg, First-principles study of the stability of fission products in uranium monocarbide, J. Nucl. Mater. 426 (1) (2012) 189-197.

[33] G. Brillant, F. Gupta, A. Pasturel, Fission products stability in uranium dioxide, J. Nucl. Mater. 412 (1) (2011) 170-176.

[34] G. Kresse, D. Joubert, From ultrasoft pseudopotentials to the projector augmented-wave method, Phys. Rev. B 59 (3) (1999) 1758.

[35] G. Kresse, J. Furthmüller, Efficiency of ab-initio total energy calculations for metals and semiconductors using a plane-wave basis set, Comput. Mater. Sci. 6 (1) (1996) 15-50.

[36] G. Kresse, J. Furthmüller, Efficient iterative schemes for ab initio total-energy calculations using a plane-wave basis set, Phys. Rev. B 54 (16) (1996) 11169.

[37] G. Kresse, J. Hafner, Ab initio molecular-dynamics simulation of the liquidmetal-amorphous-semiconductor transition in germanium, Phys. Rev. B 49 (20) (1994) 14251.

[38] H.J. Monkhorst, J.D. Pack, Special points for Brillouin-zone integrations, Phys Rev. B 13 (12) (1976) 5188

[39] M. Methfessel, A. Paxton, High-precision sampling for Brillouin-zone integration in metals, Phys. Rev. B 40 (6) (1989) 3616.

[40] N. Curry, An investigation of the magnetic structure of uranium nitride by neutron diffraction, Proc. Phys. Soc. 86 (6) (1965) 1193.

[41] Q. Yin, A. Kutepov, K. Haule, G. Kotliar, S.Y. Savrasov, W.E. Pickett, Electronic correlation and transport properties of nuclear fuel materials, Phys. Rev. B 84 (19) (2011) 195111

[42] P. Norton, R. Tapping, D. Creber, W.L. Buyers, Nature of the $5 \mathrm{f}$ electrons in uranium nitride: a photoelectron spectroscopic study of $\mathrm{UN}, \mathrm{U}, \mathrm{UO}_{2}, \mathrm{ThN}$, and Th, Phys. Rev. B 21 (6) (1980) 2572.

[43] A.E. Thompson, C. Wolverton, First-principles study of noble gas impurities and defects in uo 2, Phys. Rev. B 84 (13) (2011) 134111.

[44] H. Knott, G. Lander, M. Mueller, O. Vogt, Search for lattice distortions in UN, UAs, and USb at low temperatures, Phys. Rev. B 21 (9) (1980) 4159.

[45] H. Matzke, Science of Advanced Lmfbr Fuels, 1986.

[46] W. Sun, I. Di Marco, P. Korzhavyi, A lda + u and lda + dmft study of uranium mononitride: from nonmagnetic to paramagnetic and ferromagnetic, MRS Proceedings 1683 (2014) mrss14-1683, Cambridge Univ Press.

[47] S. Hayes, J. Thomas, K. Peddicord, Material property correlations for uranium mononitride: ii. mechanical properties, J. Nucl. Mater. 171 (2-3) (1990) $271-288$.

[48] R.A. Evarestov, A.V. Bandura, M. Losev, E. Kotomin, Y.F. Zhukovskii, D. Bocharov, A first-principles dft study of un bulk and (001) surface: comparative lcao and pw calculations, J. Comput. Chem. 29 (13) (2008) 2079-2087.

[49] C. Varvenne, F. Bruneval, M.-C. Marinica, E. Clouet, Point defect modeling in materials: coupling ab initio and elasticity approaches, Phys. Rev. B 88 (2013) 134102.

[50] D. Simonovic, M.H.F. Sluiter, Impurity diffusion activation energies in Al from first principles, Phys. Rev. B 79 (2009) 054304. 